\title{
Awareness of disabilities in pharmacy program recruitment material: are we doing enough?
}

\section{Exploring disability awareness and accessibility in pharmacy program recruitment material}

\section{- Farhat Naz HUSSAIN ${ }^{1}$, Alesha SMITH ${ }^{2}$, Kyle John WILBY ${ }^{2}$}

${ }^{1}$ College of Pharmacy, QU Health, Qatar University ${ }^{2}$ School of Pharmacy, University of Otago

\section{INTRODUCTION}

- Health professional schools are attempting to attract the best student candidates around the world

- Limited information is available on how they are working to draw interest to professional pharmacy programs, including students with disabilities

\section{AIM}

- Explore visibility of disabilities within online recruitment material for pharmacy programs

- Determine location of targeted information available to prospective students with disabilities.

\section{METHODS}

1. Digital search conducted of 'QS World University Rankings' under the subject 'Pharmacy and Pharmacology'

2. Top 50 pharmacy schools offering a professional, entry-to-practice pharmacy program selected. Websites not in English were excluded.

3. Data extraction completed by one investigator and validated by another

\section{NEXT STEPS}

1. Programs must exercise greater efforts to support recruitment of students with disabilities by including visual, audio, or written information

2. Future research should focus on whether this has an impact on student interest and admissions in pharmacy

\section{RESULTS and DISCUSSION}

- 41 out of 50 pharmacy schools met required inclusion criteria

- No institutions displayed visual student disabilities in pictures or videos of recruitment material

- $88 \%$ of institutions provide information for prospective students with disabilities

- Type of information provided was highly variable across institutions e.g. student support, non-discrimination policies and admission requirements

- $85 \%$ of top 50 pharmacy schools in the USA have information on student disability through the pharmacy homepage

- Interestingly, $62.5 \%$ of schools in Asia did not provide student disability information

- Recruitment material for pharmacy degree programs should be current, inclusive, and reflective of student populations eligible to be admitted

\begin{tabular}{|c|c|c|c|}
\hline $\begin{array}{l}\text { Universities } \\
\text { by region }\end{array}$ & $\begin{array}{l}\text { Disability in } \\
\text { pictures or } \\
\text { videos } \\
n(\%)\end{array}$ & $\begin{array}{c}\text { Disability } \\
\text { information on } \\
\text { pharmacy page } \\
n(\%)\end{array}$ & $\begin{array}{c}\text { Disability } \\
\text { information on } \\
\text { university page } \\
n(\%)\end{array}$ \\
\hline \multicolumn{4}{|l|}{ North America } \\
\hline Canada $(n=4)$ & 0 & $2(50)$ & $2(50)$ \\
\hline USA $(n=13)$ & 0 & $11(85)$ & $2(15)$ \\
\hline \multicolumn{4}{|l|}{ Europe } \\
\hline UK $(n=7)$ & 0 & $2(29)$ & $5(71)$ \\
\hline Italy $(n=2)$ & 0 & $1(50)$ & $1(50)$ \\
\hline Germany $(n=1)$ & 0 & 0 & $1(100)$ \\
\hline Spain $(n=1)$ & 0 & 0 & $1(100)$ \\
\hline Belgium $(n=1)$ & 0 & 0 & $1(100)$ \\
\hline \multicolumn{4}{|l|}{ Oceania } \\
\hline Australia $(n=3)$ & 0 & $2(67)$ & $1(33)$ \\
\hline New Zealand $(n=1)$ & 0 & 0 & $1(100)$ \\
\hline \multicolumn{4}{|l|}{ Asia } \\
\hline Singapore $(n=1)^{*}$ & 0 & 0 & 0 \\
\hline South Korea $(n=3)^{*}$ & 0 & 0 & $2(67)$ \\
\hline Hong Kong $(n=1)^{*}$ & 0 & 0 & 0 \\
\hline China $(n=3)^{*}$ & 0 & 0 & $1(33)$ \\
\hline
\end{tabular}

